\title{
ON THE FRATTINI SUBGROUP
}

\section{JOHN COSSEY AND ALICE WHITTEMORE ${ }^{1}$}

1. Introduction. Let $F$ be a free group, $R$ a normal subgroup of $F$ and $S$ a fully invariant subgroup of $R$. A question which has attracted some attention recently asks what is the structure of $F / S$ (see for example [1], [2], [3]). In this paper we shall be concerned with the Frattini subgroup of $F / S$. Recall that the Frattini subgroup $\Phi(G)$ of a group $G$ is the intersection of all the maximal subgroups of $G$; following Gaschutz [5], $G$ will be called $\Phi$-free if $\Phi(G)=1$. Our main result will be

THEOREM 1.1. If $F / R$ is residually finite ${ }^{2}$ then $F / R^{\prime}$ is $\Phi$-free.

If $G / N$ is $\Phi$-free then $\Phi(G) \leqq N[5]$, hence the following corollary is an immediate consequence of Theorem 1 .

CoROllary 1.2. If $F / R$ is residually finite and $S$ is a fully invariant subgroup of $R$ such that $S \leqq R^{\prime}$ then $\Phi(F / S) \leqq R^{\prime} / S$.

In order to obtain more precise information we must make some assumptions on $S$. This is done most conveniently in the language of varieties of groups. A variety is a class of groups closed under the operations of taking cartesian products, subgroups and homomorphic images. For a variety $\mathfrak{B}$ and an arbitrary group $G$ we obtain a fully invariant subgroup $V(G)$ of $G$, namely the intersection of all normal subgroups $N$ of $G$ such that $G / N \in \mathfrak{B}$; it is easily seen that if $\mathfrak{U}$ and $\mathfrak{B}$ are varieties with $\mathfrak{U} \leqq \mathfrak{B}$ then $\mathfrak{U}(G) \geqq \mathfrak{B}(G)$. If $X$ is a free group then $X / V(X)$ is called relatively free in the variety $\mathfrak{B}$. If $\mathfrak{U}$ and $\mathfrak{B}$ are varieties, and we denote by $\mathfrak{U} \mathfrak{B}$ the class of all groups $G$ such that $G$ has a normal subgroup $N$ with $N \in \mathfrak{U}$ and $G / N \in \mathfrak{B}$, then $\mathfrak{U} \mathfrak{B}$ is a variety and $U V(G)=U(V(G))$. We say that a variety is nilpotent if it consists of nilpotent groups and a variety has exponent 0 if it contains $\mathfrak{A}$, the variety of all abelian groups. With these remarks we can now state

THEOREM 1.3. If $F / R$ is residually finite and $\mathfrak{N}$ is a nilpotent variety of exponent 0 , then $\Phi(F / N(R))=R^{\prime} / N(R)$.

Received by the editors June 24, 1968.

1 The first author is a Fulbright-Hays scholar; he also acknowledges support from the National Science Foundation.

2 If $\mathfrak{C}$ is a class of groups, we say that a group is residually $\mathfrak{C}$ if the intersection of all normal subgroups with quotient in $\mathbb{C}$ is trivial. 
Now suppose that $\mathfrak{B}=\mathfrak{B}_{1} \mathfrak{B}_{2} \cdots \mathfrak{B}_{n}$, with each $\mathfrak{B}_{i}$ nilpotent. From Theorem 2 of Gilbert Baumslag [2] and the fact that relatively free nilpotent groups are residually finitely generated [8, Theorem 17.41], and that finitely generated nilpotent groups are residually finite [7, Theorem 3], we can deduce that $F / V(F)$ is residually finite. Moreover if $\mathfrak{B}_{1}$ has exponent 0 and $R=V_{2} \cdots V_{n}(F)$ then since $F / R$ is residually finite and $V(F)=V_{1}(R)$ we may apply Theorem 1.3 to give

CoRollaRy 1.4. If $\mathfrak{B}=\mathfrak{B}_{1} \mathfrak{B}_{2} \cdots \mathfrak{B}_{n}$ with $\mathfrak{B}_{i}(1 \leqq i \leqq n)$ nilpotent and $\mathfrak{B}_{1}$ of exponent 0 then $\Phi(F / V(F))=R^{\prime} / V(F)$. In particular $\Phi(F / V(F))$ is nilpotent.

A group $G$ is polynilpotent if it has a series $1=N_{0}<N_{1}<\ldots$ $<N_{r}=G$ with each $N_{i}$ normal in $G$ and $N_{i+1} / N_{i}$ nilpotent. With $\mathfrak{B}$ as in Corollary 1.4, $F / V(F)$ is polynilpotent. The nilpotency of its Frattini subgroup contrasts strongly with the general situation for finitely generated polynilpotent groups (see P. Hall [6, Theorem 2]).

If $\mathfrak{B}=\mathfrak{A}^{n}$ for some $n$ then $F / V(F)$ is called a free soluble group and as a special case of Corollary 1.4 we have

COROLlaRy 1.5. Free soluble groups are $\Phi$-free.

There are many other varieties $\mathfrak{B}$ for which we can determine the Frattini subgroup of $F / V(R)$, using the techniques employed in the proofs of the previous theorems. As a sample and a contrast to Theorem 1.3 we prove

THEOREM 1.6. Let $F / R$ be residually finite and let $\mathfrak{U}$ be the variety of all center extended by metabelian groups. ${ }^{3}$ Then $\Phi(F / U(R))=R^{\prime \prime} / U(R)$.

2. The proof of Theorem 1.1. Let $A$ and $B$ be finite groups and let $K$ be the group of all functions from $B$ to $A$ with coordinatewise multiplication. We define an action of $B$ on $K$ by $f^{b}\left(b^{\prime}\right)=f\left(b^{\prime} b^{-1}\right)$ for $f \in K$ and $b, b^{\prime} \in B$. With this action we can form the splitting extension of $K$ by $B$; this extension is called the wreath product of $A$ and $B$ and denoted by $A$ wr $B$. $A$ is called the bottom group, $B$ the top group and $K$ the base group of $A$ wr $B$.

The Fitting subgroup $F(G)$ of a finite group $G$ is the maximal normal nilpotent subgroup of $G$. Since the Frattini subgroup of a finite group is nilpotent, we have $\Phi(G) \leqq F(G)$.

We say that $H$ is a Hall subgroup of a finite group $G$ if its order and index are coprime.

3 That is, all groups in which the second derived group is contained in the center. 
Lemma 2.1. Let $N$ be a normal abelian Hall subgroup of the finite group $G$. Then $F(G)$ is contained in the centralizer $C_{G}(N)$ of $N$ in $G$.

Proof. $N \leqq F(G)$ by the definition of $F(G)$. Since $N$ is a Hall subgroup of $G$ and $F(G)$ is the direct product of its Sylow subgroups we have $F(G)=N \times Q$ and the result follows.

Lemma 2.2 (John Campbell [4, Theorem 8.2 (c)]). If $N$ is a normal nilpotent Hall subgroup of $G$ then $\Phi(G)=\Phi(N) \times Q$ where $(|N|,|Q|)=1$.

Let $G$ be a group, D a class of groups and $\Lambda_{\supset}$ a subset of the set of all homomorphisms from $G$ into groups in $D$. Then we say that $\Lambda_{\mathscr{D}}$ discriminates $G$ if for every finite set $g_{1}, \cdots, g_{l}$ of nontrivial elements of $G$ there exists a $\phi \in \Lambda_{D}$ such that $g_{i} \phi \neq 1(1 \leqq i \leqq l)$.

We shall assume familiarity with the paper of Gilbert Baumslag [2]. For convenience we restate Theorem 1.1: If $F$ is a free group, $R$ a normal subgroup of $F$ with $F / R$ residually finite, then $\Phi\left(F / R^{\prime}\right)=1$.

Let $\mathfrak{C}$ be the set of finite homomorphic images of $F / R$ and put $D=\left\{Z_{p}\right.$ wr $G ; G \in \mathbb{C}, p$ a prime and $\left.p \nmid|G|\right\}$. Then

LEMmA 2.3. There exists a set $\Lambda_{\mathfrak{D}}$ which discriminates $F / R^{\prime}$ such that for any $\phi \in \Lambda_{\mathcal{D}}$ with $\phi: F / R^{\prime} \rightarrow Z_{p}$ wr $G$ the image $\left(R / R^{\prime}\right) \phi$ is contained in the base group of $Z_{p}$ wr $G$.

The proof of Lemma 2.3 follows the proof of Theorem 1 of Gilbert Baumslag, using the fact that if $A$ is a free abelian group then the set of all homomorphisms from $A$ into elements of $\left\{Z_{p}, p \in \pi\right.$, with $\pi$ any infinite set of primes $\}$ discriminates $A$. The details are left for the reader.

For any group $G$ with normal subgroup $N$ we have $\Phi(G / N)$ $\geqq \Phi(G) N / N$; hence it is enough to show that for an arbitrary nontrivial element $g$ in $F / R^{\prime}$ there exists a homomorphism $\phi \in \Lambda_{\triangleright}$ such that $g \phi \notin \Phi\left(\left(F / R^{\prime}\right) \phi\right)$. The proof divides into two cases, $g \notin R / R^{\prime}$ and $g \in R / R^{\prime}$; we treat both cases simultaneously. Let $g \in F / R^{\prime}$, $g \notin R / R^{\prime}$ and let $1 \neq h \in R / R^{\prime}$. Since the centralizer of $R / R^{\prime}$ in $F / R^{\prime}$ is $R / R^{\prime}$ [1, Theorem 1], there exists an element $f \in R / R^{\prime}$ such that $[g, f] \neq 1$. By Lemma 2.3 there exists $\phi: F / R^{\prime} \rightarrow Z_{p}$ wr $G$ such that $[g, f] \phi \neq 1 \neq h \phi$. Note that if $H=\left(F / R^{\prime}\right) \phi$ and $K$ is the base group of $Z_{p}$ wr $G$, then $H \cap K$ is a normal abelian Hall subgroup of $H$ and $f \phi$, $h \phi \in H \cap K$. Since $[g, f] \phi \neq 1 g \phi \notin C_{H}(H \cap K)$ and so by Lemma $2.1 g \phi$ is not in the Fitting subgroup of $H$ and thus not in $\Phi(H)$. Moreover since $H \cap K$ is an elementary abelian $p$ group, $\Phi(H \cap K)=1$; hence by Lemma $2.2 h \phi \notin \Phi(H)$. This completes the proof of the theorem. 
3. Proofs of Theorems 1.3 and 1.6. A subset $T$ of a group $G$ is called omissible in $G$ if whenever $g p\{T, S\}=G$ for some subset $S$ of $G$ we have $g p\{S\}=G$. We need the following facts.

3.1. If $T$ is omissible in $G$ then $T \leqq \Phi(G)$.

3.2. If $T$ is a normal subgroup of $G$ and $H$ is a subgroup of $G$ with $T$ omissible in $H$ then $T$ is omissible in $G$ [4, Theorem 3.1.1].

3.3. If $G$ is nilpotent then $G^{\prime}$ is omissible in $G[4,3.3 .1$ Corollary].

Proof of Theorem 1.3. It follows immediately from Corollary 1.2 that $\Phi(F / N(R)) \leqq R^{\prime} / N(R)$. On the other hand, $R^{\prime} / N(R)$ is omissible in $R / N(R)$ (3.3) and hence in $F / N(R)$ (3.2) giving that $R^{\prime} / N(R)$ $\leqq \Phi(F / N(R))(3.1)$.

Proof of Theorem 1.6. Since $F / R$ is residually finite we have $F / R^{\prime}$ residually finite by $[2$, Theorem 2$]$, and so by Theorem 1.1 $F / R^{\prime \prime}$ is $\Phi$-free, that is $\Phi(F / U(R)) \leqq R^{\prime \prime} / U(R)$. On the other hand, $R^{\prime} / U(R)$ is nilpotent and as in the proof of Theorem 1.3, $R^{\prime \prime} / U(R)$ $\leqq \Phi(F / U(R))$.

\section{REFERENCES}

1. M. Auslander and R. C. Lyndon, Commutator subgroups of free groups, AmerJ. Math. 77 (1955), 929-931.

2. Gilbert Baumslag, Wreath products and extensions, Math Z. 81 (1963), 286-299.

3. G. Baumslag and K. W. Gruenberg, Some reflections on cohomological dimension and freeness, J. Algebra 6 (1967), 394-409.

4. John Campbell, The Frattini subgroup, Thesis, Australian National University, 1967.

5. W. Gaschutz, Über die $\Phi$-Untergruppen endlicher Gruppen, Math. Z. 58 (1953), 160-170.

6. P. Hall, The Frattini subgroups of finitely generated groups, Proc. London Math. Soc. (3) 11 (1961), 327-352.

7. K. A. Hirsch, On infinite soluble groups. (IV), J. London Math. Soc. 27 (1952), 81-85.

8. Hanna Neumann, Varieties of groups, Ergebnisse der Mathematik und ihrer Grenzgebiete, Bd 37, Springer, Berlin, 1967.

Graduate Center, City University of New York and Hunter College of the City University of New York 